\title{
CORRIGENDUM
}

\section{Local insulin-like growth factor-I of ventral prostate was upregulated during long-term castration and may function through the autocrine system}

F Gao, M Yang, C Li Luo and XH Wu

Prostate Cancer and Prostatic Diseases (2011) 14, 278; doi:10.1038/pcan.2011.20

Correction to: Prostate Cancer and Prostatic Diseases (2011) 14, 136-142; doi:10.1038/pcan.2011.8
In this article, published online 15 March 2011, the third author's name appeared incorrectly. The name should have been listed as CL Luo. 